Двас Александр Григорьевич

аспирант кафедры логистики и управления цепями поставок Санкт-Петербургского государственного экономического университета

\section{О НЕОБХОДИМОСТИ СОВМЕСТНОЙ РЕАЛИЗАЦИИ ПРОЦЕССНОГО И СИСТЕМНОГО ПОДХОДОВ ПРИ УПРАВЛЕНИИ ЛОГИСТИЧЕСКИМИ РИСКАМИ ПРОИЗВОДСТВЕННОГО ПРЕДПРИЯТИЯ}

\begin{abstract}
Аннотация:
В статье обосновывается необходимость придания детерминированного характера категориально-понятийному аппарату теории и методологии управления логистическими рисками в целях повышения эффективности функционирования логистической подсистемы производственного предприятия. Рассматриваются различные подходы к определению понятия логистического риска, в том числе произведено сравнение российских и зарубежных школ, изучающих вопросы логистических рисков. Выполнен анализ методологий выделения классификационных признаков отнесения рисков к логистическим, по результатам которого сделан вывод об отсутствии единообразия классификационных признаков и обоснований применяемых принципов классификации, а также объяснена целесообраз ность в целях решения задачи повышения эффективности функционирования логистической подсистемы производственного предприятия сконцентрировать внимание исключительно на имманентных логистических рисках, реализуя при этом совместно процессный и системный подходы к анализу логистической системы производственного предприятия, в том числе логистических издержек возникающих в процессе его деятельности.
\end{abstract}

Ключевые слова:

логистика, логистические риски, определение рисков, классификация рисков, производственное предприятие, процессный подход, системный подход.
Dvas Aleksandr Grigorievich

PhD student, Logistics and Supply Chain Management Department, Saint Petersburg State University of Economics

\section{CONCERNING THE NEED OF THE JOINT IMPLEMENTATION OF PROCESS AND SYSTEM APPROACHES WHILE MANAGING LOGISTIC RISKS OF MANUFACTURING ENTERPRISE}

Summary:

The paper substantiates the deterministic nature of theoretical and methodological categories and concepts of logistic risks management in order to improve the functioning of a logistic subsystem of manufacturing enterprise. Various approaches to the definition of logistic risk are considered. They include a comparative analysis of Russian and foreign studies reviewing logistic risks. The paper analyzes criteria methods for defining risks as logistic ones. The author concludes that there are no unified classification criteria and justification of classifying principles applied. Besides, the research explains that in order to improve the functioning of a logistic subsystem of manufacturing enterprise, it is necessary to focus exclusively on the inherent logistic risks. In this context, one should jointly implement the process and system approaches to the analysis of manufacturing enterprise logistics system, including the logistic expenses arising in the course of its activity.

Keywords logistics, logistic risks, risk identification, risk classification, manufacturing enterprise, process approach, system approach.

Как было ранее обосновано автором [1], с логистической точки зрения в наиболее общем виде деятельность любого предприятия может быть представлена следующей последовательностью агрегированных (укрупненных) технологических процессов (рис. 1).

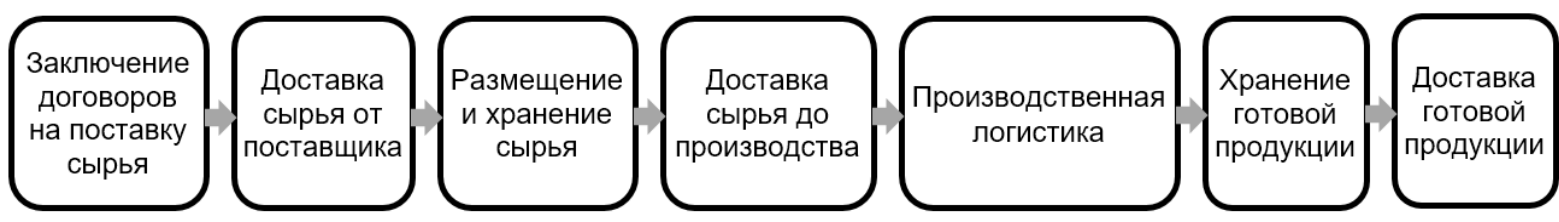

Рисунок 1 - Схема технологических процессов

Каждый из представленных технологических процессов соответствует возникновению и/или трансформации товаропотоков, что в свою очередь происходит под воздействием тех или иных логистических рисков или порождает таковые.

Поскольку по данным различных исследователей в начале XXI в. доля расходов, связанных с продвижением товара (включая затраты на хранение, транспортировку, упаковку и другие 
операции), составляет от 60 до 75 \% от стоимости продукта, попавшего к конечному потребителю, изучение вопроса логистических рисков играет важную роль в построении оптимальной структуры цепей поставок [2]. С учетом того что разные риски требуют применения различающихся методов их предотвращения или снижения, которые с неизбежностью увеличивают издержки, с той или иной степенью условности относимые к логистическим, можно сделать вывод, что категориально-понятийный аппарат теории и методологии управления логистическими рисками должен иметь детерминированный характер, обеспечивающий четкое соответствие каждого логистического риска адекватному ему методу управления.

Вместе с тем в настоящее время существует множество подходов к изучению логистических рисков, однако общепринятый подход к определению данной категории отсутствует. Во многих исследованиях происходит отождествление понятий «логистические риски», «риски в цепях поставок», «риски в логистике», «риски в логистической системе», однако зачастую фрактически используемые в публикациях разных авторов одинаковые словесные конструкции наделяются существенно отличающимися друг от друга значениями. Также при классификации рисков не соблюдается единообразие классификационных признаков, отсутствует обоснование применяемых принципов классификации.

При этом, хотя и с определенной долей условности, можно говорить о существовании самостоятельной российской школы исследования логистических рисков и построении на базе этих исследований систем управления рисками. Основное отличие российской школы состоит в предмете исследования.

Абсолютное большинство зарубежных исследователей рассматривают логистические риски через призму совокупности всех категорий, находящих отражение в теории рисков, в меньшей степени уделяя внимание изучению рисков в отдельных элементах логистической системы или же в логистических функциях, объединяя все риски в цепях поставок в единую оболочку. В большинстве зарубежных работ вместо термина «логистические риски» используется понятие «риски цепей поставок», и, соответственно, классификация подобных рисков рассматривается уже в рамках цепей поставок. При этом ключевыми трендами в определении категории риска являются следующие подходы:

- незапланированное (сверхнормативное) ухудшение имманентных параметров функционирования цепи поставок;

- незапланированное (сверхнормативное) изменение материальных и/или иных базовых логистических потоков (финансовых и/или информационных).

В результате в классификацию большинства зарубежных авторов попадают такие риски, как политические, социально-экономические, правовые. Так, например, согласно модели М. Кристофера [3], по источникам, способствующим возникновению рисков, риски в цепях поставок подразделяются на внешние (неуправляемые) и внутренние (управляемые). Внешние риски при этом подразделяются на риски спроса, поставок и внешней среды (природные катаклизмы, социально-политические события и пр.). Внутренние в свою очередь включают в себя риски процессов (производительность, надежность) и риски контроля (правила, регулирующие управление процессами).

П. Сингхал, Г. Агарвал и М. Миттал в своих трудах [4], обобщающих различные подходы классификации рисков в цепях поставок, приводят следующие критерии выделения рисков:

1) риски, связанные с эксплуатационными особенностями цепи поставок, - те параметры, которые нарушают функционирование цепи. К таким рискам можно отнести сбои в поставках, вызванные в том числе различными природными явлениями, ограниченность производственных мощностей;

2) риски, вызванные характеристиками рынка, - изменение цен, потребительского спроса, активность конкурентов и пр.;

3) риски, соответствующие особенностям функционирования бизнеса, - связаны с выбором стратегий ведения деятельности, например принятие решения об аутсорсинге каких-либо операций, выбор поставщиков, конфигурация цепей поставок, координация элементов цепи, а также с возможными ошибками прогнозов и искажением потоков информации, протекающих внутри цепи;

4) риски, вызванные особенностями продукта, - специфика жизненного цикла, технологически затрудненные особенности производства;

5) прочие риски - риски, оказывающие комплексное влияние на цепь поставок. К подобным рискам относятся политические риски, угрозы информационной безопасности, экологические риски, форс-мажоры и пр.

Аналогичные подходы транслируются в работах некоторых российских ученых. Так, в диссертации Т.В. Левиной [5] приводится классификация логистических рисков, исходя из воздействия фракторов внешней и внутренней среды на различные потоки в цепях поставок. Согласно данной классификации, логистические риски делятся на следующие: 
1) риски, затрагивающие материальный поток, - риски задержки поставок, отклонение объемов поставок от запланированных значений, получение продукции недолжного качества;

2) риски, связанные с финансовым потоком, - возможные ошибки в управлении инвестициями, нарушение обязательств взаиморасчетов контрагентов;

3) риски, проявляющиеся в информационном потоке, - риски получения некорректной информации о состоянии различных элементов внешней и внутренней среды, риски сбоев в работе информационных систем.

Российская школа исследователей логистических рисков берет свои истоки не в классической теории рисков и управления ими, а в теории управления транспортными и логистическими системами, дополняемой по мере необходимости элементами теории рисков и методологией управления рисками. Классическими в этом смысле можно считать работы профессора Санкт-Петербургского государственного экономического университета Н.Г. Плетневой, которая ввела разделение рисков в логистических системах на логистические и нелогистические. В своих исследованиях автор связывает логистические риски с рисками выполнения различных логистических операций: складирование, грузопереработка, транспортировка [6], а также с рисками, возникающими на различных уровнях логистического менеджмента, а к нелогистическим рискам относит «внешние по отношению к предприятию риски, вызванные природно-естественными, экологическими, политическими, рыночными, социальными причинами, и риски, характерные для любой другой сферы менеджмента, связанные с причинением вреда основным фондам административно-управленческого назначения, несчастными случаями, увольнением и смертью персонала и др.» [7].

Подобных взглядов на определение логистических рисков придерживаются многие отечественные исследователи. Так, например, К.Б. Стерлигов определяет логистические риски как объединение различных видов рисков во всех элементах логистической системы - как в процессе изменения материальных, финансовых и информационных потоков, так и в процессе управления [8].

И.В. Яхнеева в своей диссертации «Теория и методология управления рисками в системах поставок» [9], развивая методологию, предложенную Н.Г. Плетневой, обосновала, что в традиционном подходе к управлению рисками выделяются риски на уровне отдельных звеньев цепи поставок, подразделений предприятия или же более локально на уровне проектов, что в свою очередь приводит к различным негативным последствиям, так или иначе связанным с отсутствием взаимодействия между звеньями цепи в рамках управления рисками, и, соответственно, актуализируется необходимость применения системного подхода к анализу и управлению логистическими рисками в рамках всех бизнес-процессов, осуществляемых в цепи поставок. Соответственно, классификация рисков проведена указанным автором согласно системному подходу.

Обобщая выводы и результаты приведенных и ряда других публикаций, представляется целесообразным в целях решения задачи повышения эффективности функционирования логистической подсистемы производственного предприятия сконцентрировать внимание исключительно на возникающих в логистических системах логистических (в трактовке Н.Г. Плетневой) рисках, реализовав совместно процессный и системный подходы к изучению логистической системы производственного предприятия, в том числе логистических издержек, возникающих в процессе его деятельности.

\section{Ссылки:}

1. Двас А.Г. Издержки управления запасами и методы их снижения [Электронный ресурс] // Науковедение : интернетжурнал. 2016. T. 8, № 5. URL: https://naukovedenie.ru/PDF/78EVN516.pdf (дата обращения: 08.02.2018).

2. Гаджинский А. Взаимосвязь логистики и маркетинга [Электронный ресурс] // Advertology. Наука о рекламе. 2008. 13 февр. URL: http://www.advertology.ru/article57906.htm (дата обращения: 05.03.2015).

3. Christopher M. Understanding Supply Chain Risk: A Self-Assessment Workbook / Centre for Logistics and Supply Chain Management at the Cranfield School of Management. Cranfield, 2003. P. 54.

4. Singhal P., Agarwal G., Mittal M.L. Supply Chain Risk Management: Review, Classification and Future Research Directions // International Journal of Business Science and Applied Management. 2011. Vol. 6, no. 3. P. 15-42.

5. Левина Т.В. Формирование системы управления логистическими рисками в цепях поставок строительных предприятий : автореф. дис. ... канд. экон. наук. М., 2014.

6. Плетнева Н.Г. Анализ рисков логистики и цепей поставок: подход к классификации и алгоритм принятия решений // Вестник ИНЖЭКОНа. Серия: Экономика. 2006. № 4 (13). С. 213-220.

7. Там же.

8. Стерлигов К.Б. Механизмы управления рисками в логистике // Логистика \& система. 2006. № 4. С. 49-55

9. Яхнеева И.В. Теория и методология управления рисками в системах поставок : автореф. дис. ... д-ра экон. наук. Самара, 2013. $38 \mathrm{c}$.

\section{References:}

Christopher, M 2003, Understanding Supply Chain Risk: A Self-Assessment Workbook, Centre for Logistics and Supply Chain Management at the Cranfield School of Management, Cranfield, p. 54. 
Dvas, AG 2016, 'Inventory management costs and methods of reducing them', Naukovedeniye: internet-zhurnal, vol. 8, no. 5, viewed 08 February 2018, <https://naukovedenie.ru/PDF/78EVN516.pdf>, (in Russian).

Gadzhinsky, A 2008, 'The relationship between logistics and marketing', Advertology. Nauka o reklame, February 13, viewed 05 March 2018, <http://www.advertology.ru/article57906.htm>, (in Russian).

Levina, TV 2014, Development of logistics risks management system in supply chains of construction companies, PhD thesis abstract, Moscow, (in Russian).

Pletneva, NG 2006, 'Analysis of the risks of logistics and supply chains: approach to classifying and decision-making algorithm', Vestnik INZHEKONa. Seriya: Ekonomika, no. 4 (13), pp. 213-220, (in Russian).

Singhal, P, Agarwal, G \& Mittal, ML 2011, 'Supply Chain Risk Management: Review, Classification and Future Research Directions', International Journal of Business Science and Applied Management, vol. 6, no. 3, pp. 15-42.

Sterligov, KB 2006, 'Mechanisms of risk management in logistics', Logistika \& sistema, no. 4, pp. 49-55, (in Russian).

Yakhneeva, IV 2013, Theory and methods of risk management in supply chains, D.Phil. thesis abstract, Samara, 38 p., (in Russian). 